Ann. Abeille, I962, 5 (3), I75-179

\title{
NOTES ZOOGÉOGRAPHIQUES ET SYSTÉMATIQUES SUR LES BOMBIDAE
}

\author{
R. DELMAS \\ Laboratoire de Recherches de la Chaire de Zoologie, \\ Centre de Recherches agronomiques du Midi, École nationale \\ supérieure agronomique, Montpellier (Hérault).
}

L'importance reconnue des Bourdons comme agents pollinisateurs de quelques plantes cultivées, certaines légumineuses en particulier, a attiré sur ce groupe d'Apiaires l'attention des phytotechniciens qui ont envisagé leur utilisation pour augmenter le rendement des cultures de graines de semence ou les ont même utilisés comme auxiliaires pour la réalisation de croisements expérimentaux. Mais la mise au point d'une bonne technique implique le choix de l'espèce la mieux adaptée à chaque cas particulier, donc, tout d'abord, la possibilité de l'identifier correctement et ensuite la connaissance de son domaine géographique naturel ou des conditions écologiques de son habitat. C'est pour aider à la solution de ces problèmes que je publierai ici quelques notes systématiques et zoogéographiques sur ce groupe au fur et à mesure de l'avancement de la prospection entreprise.

\section{I. - IE “BOMBUS BRODMANNICUS » VOGT DES ALPES FRANÇAISES}

\section{SOMMAIRE}

L'auteur décrit le mâle encore inconnu du Bombus brodmannicus VoGT des Alpes françaises et il indique les caractères qui permettent de le distinguer dans les deux sexes de B. pyrenaeus J. PEREz. Il estime qu'il s'agit de deux espèces distinctes.

En I928, R. BËNOIST a signalé la capture en diverses régions des Alpes françaises (Alpes-Maritimes, Basses-Alpes, Hautes-Alpes, Savoie), de toute une série d'individus $q$ et $\breve{q}$ du $B$. pyrenaeus J. PEREZ où le jaune était remplacé par du blanc pur et il les a rattachés, bien qu'avec doute, à une forme décrite par $O$. VoGT en Igog du 
Caucase sous le nom de $B$. pyrenaeus var. brodmannicus. Des individus analogues existent dans la collection du Muséum national d'histoire naturelle venant des collections O. Sichei (I $q$ des Basses-Alpes), J. Perez (I $q$ des Basses-Alpes et I $\Varangle$ de

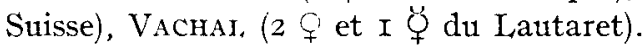

Ayant chassé pendant l'été I955 dans la région de Briançon (Htes-Alpes) j'ai pris, à mon tour quelques exemplaires de cette forme mal connue : I $q$ et I 5 sur un total d'environ 2 ooo bourdons capturés, ce qui indique sa relative rareté. Mais en sus, j'ai récolté les $\sigma^{x}$ encore inconnus qui doivent lui être attribués ( 7 individus). En I957, j'ai fait de nouvelles captures au col de Vars (attitude 2 ooo mètres), puis en I958 dans les régions de Colmars et d'Allos (I 800 à I 900 mètres). Je me propose de préciser ici les caractères qui distinguent ce brodmannicus des Alpes du vrai pyrenaeus J. PEREZ dans les deux sexes. Suivront quelques considérations sur son statut taxonomique et sa localisation géographique.

\section{FEMEI,I,ES ET OUVRIÈRES}

La différence la plus apparente entre les deux formes dans le sexe femelle, celle qui les a fait dès l'abord distinguer, réside dans la teinte des poils clairs. Si l'on excepte ceux qui recouvrent la partie dorsale des quatre derniers tergites abdominaux, tous les autres, quelle que soit leur position, sont parfaitement blancs chez brodmannicus VOGT alors que chez pyrenaeus J. PEREz ils sont d'un jaune laiton vif. Certes, chez ce dernier ils peuvent s'éclaircir chez les exemplaires ayant beaucoup volé, mais ils ne perdent pas leur nuance jatune ; en collection, à l'abri de la lumière, ils deviennent simplement un peu plus foncés en vieillissant. Jamais on ne peut hésiter entre les deux colorations.

Une différence se remarque aussi dans la répartition de ces poils clairs. Dans les deux formes, elle n'est pas exactement la même et surtout sa variabilité suit une loi différente. Les choses se voient particulièrement bien sur le $2^{\mathrm{e}}$ et le $3^{\mathrm{e}}$ tergite.

Chez le brodmannicus des Alpes, le second tergite est couvert de poils clairs sans aucun poil noir entremêlé, tandis que tous mes pyrenaeus de la même région (sousespèce tenuifasciatus VoGT de certains auteurs, $60 \nmid$ ou $\not$ examinées) en ont toujours en plus ou moins grand nombre. L,eur répartition fluctue par degrés insensibles depuis une étroite bordure caudale noire jusqu'à l'envahissement total du tergite par le noir, à l'exception de quelques poils restés jaunes à l'extrémité orale.

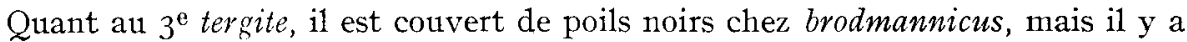
toujours cependant des poils blancs sur les parties latérales repliées et aussi, sur le disque, une invasion plus ou moins marquée de poils de même couleur. Ils y paraissent d'abord en étroite bordure au bord caudal du tergite, mais ils peuvent dans certains échantillons envahir tout le dessus où ils restent mêlés aux noirs sans former de taches bien définies. Les poils rouges y sont exceptionnels, généralement absents : au plus I à 2. Chez pyrenaeus tenuifasciatus des Alpes, les conditions sont bien différentes. L,e $3^{\mathrm{e}}$ tergite (à l'exception des parties latérales qui ont des poils jaunes) est toujours envahi par des poils rouges. Chez les individus les plus sombres, ils forment une simple bordure caudale étroite, mais chez d'autres, ils arrivent à occuper à peu près complètement tout le tergite. Il ne persiste alors, à son extrémité orale qu'une très faible bande noire. 
La comparaison avec des pyrenaeus pyrenxeus J. PEREZ de la race typique des Pyrénées apporte des résultats analogues. Cette espèce y est plus claire qu'aux Alpes en général, mais on y retrouve cependant l'envahissement du $3^{\text {e }}$ tergite par des poils rouges venant de l'arrière toujours aussi marqué : par contre, le nombre de poils noirs sur le $2^{\mathrm{e}}$ tergite est beaucoup moins important en général. Cependant je n'ai trouvé aucun individu qui n'en possède au minimum quelques unités.

Troisième différence, la plus importante certainement aux yeux d'un spécialiste du groupe : la pilosité du brodmannicus des Alpes est plus courte et moins hérissée que celle du pyrenaeus.

A côté de ces différences d'ordre morphologique se manifestent des différences d'ordre écologique. Dans la région de Briançon oì j'ai chassé en I955, pyrenxeus temuifasciatus était un animal vraiment alpin qui ne commençait à être abondant qu'au voisinage de $2000 \mathrm{~m}$. Il se rencostrait dans des prairies où il butinait presque toujours sut divers Phyteuma; brodmannicus vivait plus bas depuis $\mathrm{r} 400 \mathrm{~m}$ jusque vers I $700 \mathrm{~m}$. Il volait sur les talus pierreux des pentes faiblement boisées, butinant surtout sur Cerinthe, mais aussi sur Calamintha, Epilobium, Scabiosa et même Echium. Il m'a semblé aussi un peu plus tardif. Je l'ai retrouvé dans des conditions analogues en 1958 à Colmars et Allos (Basses-Alpes) mais à une altitude supérieure (de I 800 à $\mathrm{I} 900 \mathrm{~m}$ ).

\section{MÂLES}

Pas plus que mes prédécesseurs, je n'ai trouvé dans les Alpes de mâles à poils blancs. J'ai donc été amené à rechercher le mâle alpin encore inconnu du brodmannicus parmi ceux qui ressemblaient au vrai pyrenxeus c'est-à-dire qui avaient les mêmes genitalia et des poils jaune laiton. J'ai constaté assez vite que certains de ceux-ci que je prenais dans les localités où se rencontraient des brodmannicus $q$ étaient caractérisés par une vêture moins longue et moins hérissée. D'autre part, l'absence totale de poils noirs sur le $2^{e}$ tergite se retrouvait chez eux comme chez les $\bigcirc$ ou les $\zeta$. Par contre, les différences sur le $3^{\text {e }}$ tergite étaient moins évidentes, celui-ci étant noir dans les deux types de mâles. I a barbe des mandibules était très claire, nettement plus claire que chez les pyrenxeus tenuifasciatus en ma possession, enfin et surtout, les tibias postérieurs étaient différents. Chez brodmannicus $\sigma^{x}$, comme ches les $\sigma^{x}$ des autres Pratobombus français, la face externe des tibias postérieurs porte des soies sur toute la surface ou en est dépourvue seulement dans une aire préapicale limitée, tandis que chez pyrenaeus pyrenaeus PEREz et pyrenaeus tenuifasciatus VOGT la face externe des tibias postérieurs est dépourvue de soies, et luisante dans une aire dont la longueur et la largeur sont chacune supérieure à la moitié des dimensions correspondantes du tibia. Ceci peut être interprété comme une certaine tendance à la féminisation des tibias. moins marquée cependant que celle que l'on observé chez des Bombus str. s. (terrester, etc.). Je consid̀̀re les mâles de ce type comme ceux du brodmannicus alpin.

\section{STATUT TAXONOMIQUE}

La présence de ces deux formes voisines mais cependant morphologiquement et biologiquement séparées en des localités écologiquement un peu différentes mais voisinant à quelques centaines de mètres près, incite à les considérer comme des espè- 
ces distinctes. L'une n'est certainement pas autre chose que la race alpine du $B$. $p y$ renaeus. Quant à l'autre, elle est à peut près sûrement à rattacher au $B$. brodmannicus du Caucase que Skorikov I922 consi dère déjà comme une espèce véritable.

Il m'a été possible au cours d'une visite trop rapide au professeur O. VoGT en fin 1955 de voir les types de ce brodmannicus dans sa collection. Il m'a semblé alors, à la suite d'un examen assez superficiel, qu'ils ne présentaient pas de différences essentielles avec la forme des Alpes, en ce qui concerne les femelles. Pour les mâles ceux qui sont considérés comme typiques au Caucase ont des bandes blanches comme les femelles, mais il y existe aussi une variété à bandes jaunes représentée dans la collection VOGT. La question se pose donc de savoir si la forme des Alpes à mâles toujours jaunes doit être considérée comme une sous-espèce distincte. Je m'abstiendrai pour l'instant de prendre une position ferme sur ce point, n'ayant plus sous les yeux les types caucasiens, pour vérifier la longueur de leur vestiture et l'étendue de la plage glabre de la face externe des tibias postérieurs de leurs mâles. N'y auraitil pas d'ailleurs, au Caucase même, deux races locales distinctes différentes par leurs mâles? Notre ignorance sur ce point ne nous permet pas d'avoir une opinion sur le statut taxonomique exact des populations alpines de ce bourdon. Cependant cette existence dans les Alpes d'une forme plus ou moins voisine du vrai Bombus brodmannicus YOGT appelle un certain nombre d'autres remarques.

\section{REMARQUES ZOOGÉOGRAPHIQUES}

On affirme couramment que le type de coloration à bandes blanches associées à une région anale rouge est caractéristique des bourdons de la région caucasienne oì il se rencontre dans un grand nombre d'espèces. On l'oppose à la coloration la plus fréquente a11x Pyrénées o11 même aux Alpes où les bandes claires seraient jaunes et jamais blanches, et celà est donné comme un exemple particulièrement net d'une " convergence régionale " comme il y en a beaucoup dans le groupe. Les $q$ et $\zeta$ du brodmannicus des Alpes viennent donc constituer une exception très remarquable à cette répartition des types de coloration. Je me suis demandé s'il n'en existerait pas d'autres. Richariss I928 a bien signalé de Bourg d'Arud dans l'Isère une petite $Q$ colorée de même. I1 la considère comme un Lapidariobombus incertus Mor. mais je pense qu'il doit s'agir en réalité d'un brodmannicus, les caractères indiqués pouvant parfaitement s'y appliquer. Bien plus anciennement SichEL (I855) a signalé un bourdon qu'il considère comme une forme de silvarum L. possesseur d'une pilosité d'un blanc de neige récolté dans les Basses-Alpes par M. BÉLI.IER de la Chavignerie. J'ai retrouvé dans la collection du Muséum ce qui est peut-être 1'échantillon origina1. I1 est placé au milieu des silvarum avec une étiquette d'origine "Basses-Alpes " et de la main de Siché une deuxième «v. nivalis ", mais j'ai pu constater qu'il s'agit là aussi d'un brodmannicus. Jusqu'à nouvel ordre cette espèce paraît donc constituer aux Alpes une exception unique du point de vue de sa coloration. 


\section{SUMMARY}

\section{ZOOGEOGRAPHIC AND SYSTEMATIC NOTES ON THE Bombidae \\ I. Bombus brodmanmicus VOGT OF THE FRENCH ALPS}

It could be argued that Bumble Bees, up to now classified as pyrenaeus variety brodmannicus voGT, such as are found in the Alps and of which the writer describes above the hitherto unknown male, belong to a species distinct from pyrenaeus l'EREZ which inhabits the same area. It is highly probable that these bees are linked with brodmunicus vost which is already considered, by SKORIKOV (I922) for example, to be a distinct species. However, geographically separated from the type living in the Caucasus, it is possible that they have developed in the $A$ lps a special race possessing a monomorphic male. Only an exact knowledge of the populations inhabiting the Caucasian regions and of the significance of dimorphism in the males of these areas, which may indeed be no more than an indication of the presence of two local and distinct races, would enable one to form an opinion upon the taxonomic status of the Alpine populations of this Bumble Bee.

\section{RÉFÉRENCES BIBLIOGRAPHIQUES}

Bexorst R., I928. Etude sur la faune des Hyménoptères des Alpes françaises. Ann. Soc. Enl. France, 97, $389-417$.

RichardS O. W., 1928. Notes on aculeate IIymenotera captured in France, with the description of a new race of Crossoceris elongatulus V. de Lind. Bull. Soc. Ent. France, 218-224, 13 figures.

Sichel J., 1855. (Communications.) Bull. Soc. Ent. France, 73-74.

Skorikov A. S., 1922. Les bourdons de la faune paléarctique. P’artie I Biologie générale (la zoogéographie y compris). Bull. Stat. Rég. Prot. Plantes P'etrograd, 4, Part I. [en russe].

Voct O., Igog. Studien über das Artproblem. I. Mitteilung über das Variierden der Hummeln r. Teil. Sits. Ges. Valurf. Freunde, $28-2.4$, I pl. 